\title{
eJRIEPS
}

Ejournal de la recherche sur l'intervention en éducation physique et sport

$27 \mid 2012$

Varia

\section{L'activité didactique empêchée en EPS en milieu difficile : contribution à la théorie de l'action conjointe en didactique}

The didactic activity prevented in PE in difficult environments: contribution to the theory of joint action in teaching

Nathalie Monnier et Chantal Amade-Escot

\section{OpenEdition}

Journals

Édition électronique

URL : http://journals.openedition.org/ejrieps/3121

ISSN : 2105-0821

Éditeur

ELLIADD

Référence électronique

Nathalie Monnier et Chantal Amade-Escot, «L'activité didactique empêchée en EPS en milieu difficile contribution à la théorie de l'action conjointe en didactique », eJRIEPS [En ligne], 27 | 2012, mis en ligne le 01 juillet 2012, consulté le 20 novembre 2019. URL : http://journals.openedition.org/ejrieps/3121

Ce document a été généré automatiquement le 20 novembre 2019.

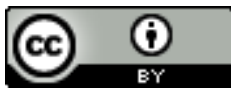

La revue eJRIEPS est mise à disposition selon les termes de la Creative Commons Attribution 4.0 International License. 


\title{
L'activité didactique empêchée en EPS en milieu difficile : contribution à la théorie de l'action conjointe en didactique
}

\author{
The didactic activity prevented in PE in difficult environments: contribution to \\ the theory of joint action in teaching
}

Nathalie Monnier et Chantal Amade-Escot

Cet article s'intéresse à ce qui s'enseigne en EPS en milieu difficile. Au point de départ de la recherche (Monnier 2009) était la volonté de porter un éclairage didactique, descriptif et compréhensif sur les constats-posés par des travaux réalisés sous différents cadrages théoriques - mettant en évidence l'affaiblissement des savoirs fondamentaux au bénéfice de savoirs plus superficiels et de savoir-faire sociaux dans les établissements de milieu difficile. Pour instruire notre question de recherche nous avons construit un protocole convoquant d'une part, le cadre théorique didactique et d'autre part, celui de la clinique de l'activité. L'ambition était d'identifier, à la lumière de ce double cadre, les enjeux en tension dans l'activité de l'enseignant au travail, afin d'éclairer l'action didactique observée et de produire des pistes explicatives rompant avec les discours d'impuissance du système ou d'accusation des enseignants.

Nous présentons tout d'abord l'ancrage théorique didactique dans lequel se situe notre étude puis les points d'appuis majeurs permettant de caractériser l'enseignement en milieu difficile. Nous traitons ensuite des conditions épistémologiques et méthodologiques que nous avons réunies pour rendre compatible les deux orientations de recherche que nous convoquons dans l'élaboration de notre système de recherche (Leutenegger 2003). Nous montrons enfin en quoi notre travail contribue en même temps qu'il s'y adosse, à la théorie de l'action conjointe telle que développée au sein du courant comparatiste en didactique. 


\section{Préambules éclairant la posture de recherche}

\section{1. Une problématique didactique}

2 Notre recherche s'inscrit dans le cadre problématique de l'approche comparatiste en didactique, qui pose les prémisses d'une réflexion visant à déterritorialiser le regard tout en en garantissant à la fois la pertinence et l'intérêt du champ didactique au sein des sciences de l'éducation, et au-delà, au sein des sciences humaines et sociales. Il s'agit, dans cette perspective, de mettre en tension les recherches des didactiques disciplinaires pour repérer et discuter, à partir des concepts majeurs des théorisations antérieures (notamment, la théorie des situations didactiques de Brousseau) ce qui relèverait de composantes spécifiques d'une part et génériques d'autre part (Schubauer-Leoni \& Leutenegger, 2003). Ce travail de comparaison des différentes didactiques mais aussi «du» didactique dans différentes institutions suppose en conséquence, des emprunts renouvelés aux autres champs des sciences humaines et sociales dont les plus marqués sont le recours aux théories des institutions et aux théories du sujet. La convocation que nous faisons des travaux de la clinique de l'activité s'inscrit dans ce processus.

Les recherches comparatistes en didactique ont créé les conditions de la construction et du développement de la théorie de l'action conjointe en didactique (TACD) (Schubauer-Leoni et al., 2007; Sensevy, 2007; Sensevy \& Mercier, 2007) sur laquelle nous prenons appui et qui «suppose l'étude et la modélisation de l'action effective de celui qui enseigne et de ceux qui apprennent dans des conditions données, avec des effets sur le système triadique ainsi que sur ses composantes" (Schubauer-Leoni \& Leutenegger, 2002, p. 233).

Dans la continuation des visées de recherche initialement posées par Guy Brousseau, le programme de recherche comparatiste en didactique envisage l'action de l'enseignant et de l'élève en classe en les examinant sous forme de jeux (Brousseau, 1988). Il s'attache notamment à décrire, à partir de l'observation de classes ordinaires, le jeu du professeur sur le jeu des élèves impliqués dans un jeu d'apprentissage (Sensevy \& Mercier, 2007). Dans cet article nous nous appuyons plus particulièrement, pour décrire l'action conjointe du professeur et des élèves, sur le triplet de genèses (Mésogenèse, Topogenèse, Chronogenèse) qui permet de rendre compte de la dynamique évolutive des interactions didactiques. Nous avons ainsi différencié les actions du professeur ou des élèves qui : i) produisent un effet sur le milieu (descripteur mésogénétique), ii) modifient la répartition des responsabilités et des moyens de contrôle en situation (descripteur topogénétique) et enfin, iii) contribuent à l'avancée du savoir (descripteur chronogénétique). Plus particulièrement, le partage des responsabilités a été envisagé à partir des moyens de contrôle qu'offre le dispositif aux élèves pour sélectionner les réponses produites dans le jeu et se rapprocher progressivement des réponses prometteuses (ou traits pertinents selon Schubauer-Leoni, 2008).

\section{2. La prise en compte des travaux sur l'enseignement en milieu difficile}

De nombreuses recherches ont mis en évidence les caractéristiques de ce qui s'enseigne en milieu difficile. Dans le cadre de cet article nous pointons rapidement, à partir des principaux résultats des travaux de la sociologie de l'éducation et de quelques autres 
plus rares en didactique sur ce sujet, les aspects qui permettent de repérer le particularisme des pratiques enseignantes dans en contextes difficiles. Ainsi, il a été repéré que les difficultés rencontrées par les enseignants pour faire cours les amènent à mettre en concurrence les visées de maintien de l'ordre et d'apprentissage (van Zanten, 2001; Kherroubi \& Rochex, 2002, 2004). Cette mise en concurrence peut se traduire ou s'exprimer par le souci de limiter les risques de conflits avec les élèves, risques accrus lorsque les élèves buttent, voire sont en échec dans l'apprentissage proposé. Les travaux de l'équipe DIDIREM indiquent que cette préoccupation conduit les enseignants à réduire leurs exigences, à retarder le moment de la confrontation de l'élève à la complexité des savoirs, à tenter d'obtenir des réussites à moindre coût par simplification exagérée des tâches, autant de stratégies relevant de sous-ajustements didactiques. De la même manière, la crainte des dérapages lors des moments d'échanges en grand groupe, est considérée être en partie à l'origine de la disparition des phases d'institutionnalisation, c'est-à-dire les phases au cours desquelles l'enseignant rend explicite et officiel à l'ensemble des élèves le savoir appris ou censé être appris (Butlen, Peltier-Barbier, \& Pezart, 2002, Peltier-Barbier, 2004). Globalement les auteurs soulignent l'existence de sous-ajustements didactiques, de scénarios didactiques à risques minima et de malentendus se traduisant par un affaiblissement des savoirs enseignés dans ces milieux (Bautier \& Rochex, 1997 ; Poggi-Combaz, 2002 ; Rochex, 1997).

Notre étude a tenté d'accéder aux raisons de ces constats, incontestables du point de vue d'où ils sont posés, afin d'initier des pistes explicatives tout en rompant avec l'idée volontariste (parfois soutenue par les connaissances produites par la recherche sur les élèves et l'enseignement en milieu difficile) qui considère que les enseignants devraient faire mieux et plus, ou tout du moins, qui stigmatise leur renoncement supposé.

\section{3. De la difficulté des enseignants au travail à la psychologie du travail}

4 L'intensité et la fréquence d'expression du sentiment de difficulté ou d'échec dans les discours des enseignants exerçant leur métier en milieu difficile nous ont amené à nous intéresser aux travaux développés, au sein de la psychologie du travail, par le courant de «la clinique de l'activité ». Cette orientation de recherche, développée par Clot $(1999,2001)$ considère en s'appuyant sur les théorisations de Vygotski et Bakhtine, que l'activité réalisée n'est pas toute l'activité du sujet mais «l'actualisation d'une des activités réalisables dans la situation où elle voit le jour " (Clot, 2001, p. 10). L'action, observable, n'est que l'état momentané de résolution des conflits du réel qui se jouent entre tous les possibles, la solution qui a vaincu dirait Vygotski (cité par Clot, 2001). Le réel de l'activité c'est donc ce qui se fait mais aussi « ce qui ne s'est pas fait », « ce qui n'a pas pu se faire ", « ce qu'on fait pour ne pas faire ce qui est à faire » (Clot, 1999). Est ainsi mise en évidence la part "contrariée », "rentrée ", "impossible», ou encore "empêchée » de l'activité de l'homme au travail. Le concept "d'activité empêchée » permet d'incorporer les conflits du réel, le possible ou l'impossible, à l'activité. Il fonde, en même temps qu'il l'explique, à la fois le développement de l'activité (évolution des buts, motifs et mobiles portés par la recherche d'efficience mais aussi par le sens de l'action) et sa mise en souffrance (pour un développement voir Clot, 1999).

Nous pensons que l'approche développée par la clinique de l'activité constitue un point de vue porteur d'éléments de compréhension des effets des milieux difficiles sur 
l'action didactique des enseignants en classe. Nous faisons nôtre en particulier, l'éclairage spécifique, théoriquement encadré, que cette orientation en psychologie du travail permet de produire sur le sujet enseignant (Saujat, 2004).

Par extension, nous avons considéré heuristique de problématiser l'activité professorale en terme "d'activité didactique empêchée" (Pour une discussion, Monnier, 2008, Monnier \& Amade-Escot, 2007) - afin de décrire et d'analyser ce qui s'enseigne/s'apprend en classe en s'attachant à la fois à la part réalisée et à la part empêchée de l'activité. Nous postulons que l'articulation au sein de la TACD d'une problématique relevant de l'activité empêchée donne accès à une compréhension générique de l'action didactique des enseignants.

\section{2. Élaboration du système de recherche mis en place}

5 Le recours à des concepts tirés de la clinique de l'activité a imposé un travail de conversion méthodologique des protocoles usuels issus des deux traditions de recherches convoquées : la didactique et la psychologie du travail.

\section{1. Les conversions opérées sur les outils empruntés à la clinique de l'activité}

Dans cette théorisation, nous l'avons brièvement évoqué, l'activité réalisée ne rend pas compte de toute l'activité déployée par le sujet pour réaliser la tâche. Ainsi, accéder au réel de l'activité, mettre au jour l'activité empêchée, les possibles non réalisés, ne peut pas se faire par la seule observation de l'action. Il est nécessaire d'emprunter des voies indirectes, c'est-à-dire dialogiques. Le dialogue mis en place par le chercheur constitue une nouvelle situation dans laquelle une autre des activités réalisables peut, potentiellement, être actualisée. Le sujet se confronte par l'intermédiaire de l'autre, celui à qui il s'adresse, à sa propre activité, c'est en cela que l'on peut dire qu'il s'agit d'auto-confrontation. Les ergonomes ont élaboré diverses techniques d'entretien d'auto-confrontation, parmi elles nous avons retenu la méthode d'instruction au sosie qui dessine le cadre du dialogue comme un jeu de rôle entre le travailleur et une autre personne désignée comme son sosie (Oddone, Rey \& Briante, 1981; repris par Clot 1999). Le protocole méthodologique consiste à faire raconter par le travailleur ce que son sosie doit savoir pour être en mesure de le remplacer dans son travail. Chez Clot l'instruction se déroule à l'occasion d'un entretien filmé entre le travailleur et le chercheur (le sosie) consécutivement à la lecture de la consigne d'instruction. Dans un deuxième temps, le travailleur réagit au visionnage du film de cet entretien.

Nous retenons du fondement théorique de la méthode d'instruction au sosie que deux éléments fondamentaux circonscrivent le contexte de « réalisation » de son activité par le sujet: A propos de quoi, parmi la diversité des tâches qui lui incombe, faire parler le travailleur? À qui le faire s'adresser? Nous avons pris, en répondant à ces questions, des options méthodologiques qui permettent le traitement de notre problématique de recherche dans la perspective de l'approche comparatiste en didactique. 


\section{1. 1. De quels aspects du métier parler : la consigne d'instruction}

7 Pour « faire parler le métier ", des enseignants d'EPS en milieu difficile, nous avons tout d'abord transformé la consigne initiale d'Oddone qui est concise et large (Oddone et al. 1981), en une série de questions-guide amenant l'enseignant qui a réalisé la séance (filmée par ailleurs par le chercheur) à faire un bilan réflexif sur celle-ci en évoquant tout d'abord son thème, l'objectif visé, les dispositifs mis en place, les productions des élèves. Puis, en tenant compte de ce qui s'est passé, en précisant ce qui pourrait être envisagé pour la séance suivante, celle qui sera hypothétiquement prise en charge par le sosie. Enfin, l'enseignant est invité à aborder les éléments liés à la conduite de la classe (habitudes de fonctionnement, rituels, attitudes vis à vis des comportements déviants notamment).

\section{1. 2. A qui faire s'adresser l'enseignant observé : le choix du sosie}

8 Nous avons opté pour un sosie qui ne soit pas le chercheur mais un autre enseignant d'EPS exerçant en établissement de centre-ville afin que ce dernier soit, en quelque sorte, étranger à la situation de travail en milieu difficile. En ne partageant pas la même vision du réel que l'enseignant observé, nous considérons que le sosie (lors de l'échange avec l'enseignant observé), permet à l'activité de sortir de l'implicite d'un contexte qui est très naturalisé.

\section{1. 3. Déroulement de l'instruction au sosie}

Dans notre recherche, le protocole d'instruction au sosie devient un ensemble articulé d'entretiens (voir schéma 1, infra) qui occasionne trois temps d'auto-confrontation, et donc, au sens fort du terme, de «ré-alisation de son activité » par l'enseignant observé :

- un premier temps demande à l'enseignant observé de rédiger l'instruction à l'attention de son sosie après la séance filmée, en s'appuyant sur le questionnaire guide fourni par le chercheur.

- un deuxième temps, au cours duquel le contexte de réalisation est dessiné par le dialogue ( entretien-sosie ») entre l'enseignant observé et son sosie. Cet entretien se déroule en présence du chercheur, mais il est à l'initiative du sosie qui a pris connaissance de l'instruction rédigée par l'enseignant observé (bilan de séance à chaud). Soulignons que le sosie n'a pas vu la séance. Lors de cet entretien le chercheur intervient, en fin d'entretien, en réaction à ce qui se dit, pour prolonger une question ou faire préciser une réponse.

- un dernier temps, que nous appelons «entretien post-protocole», se déroule entre l'enseignant observé et le chercheur à partir des traces de l'entretien-sosie (retranscription verbatim de l'enregistrement). Dans ce dernier temps, éloigné de plusieurs semaines de l'entretien-sosie, l'enseignant est tout d'abord sollicité, pour évoquer de mémoire les points de l'entretien-sosie qui l'ont intéressé, questionné, ou peut-être même, troublé. Ensuite, le chercheur lui demande de réagir à des extraits de cet entretien qu'il a retenu sur la base des analyses didactiques effectuées à partir de la vidéo de la séance observée, ce qui explique l'espacement entre les deux entretiens (pour un développement, Monnier \& Amade-Escot, 2007). 


\section{2. Intégration de l'instruction-sosie au protocole d'observation du didactique ordinaire} (2003) articule des enregistrements vidéo de séances et des entretiens. Se déroule tout $\mathrm{d}$ 'abord un entretien ante permettant de s'entendre avec l'enseignant sur la séance ou le dispositif expérimental, puis la séance de travail en classe est filmée et, enfin, des entretiens post avec l'enseignant et/ou les élèves sont effectués. Les entretiens, dans ce dispositif, jouent le rôle de discutant des phénomènes observés sur la bande. L'option prise par Leutenegger est de rentrer dans l'analyse par la séance de travail en classe.

Schéma 1. Dispositif de recherche mis en œuvre

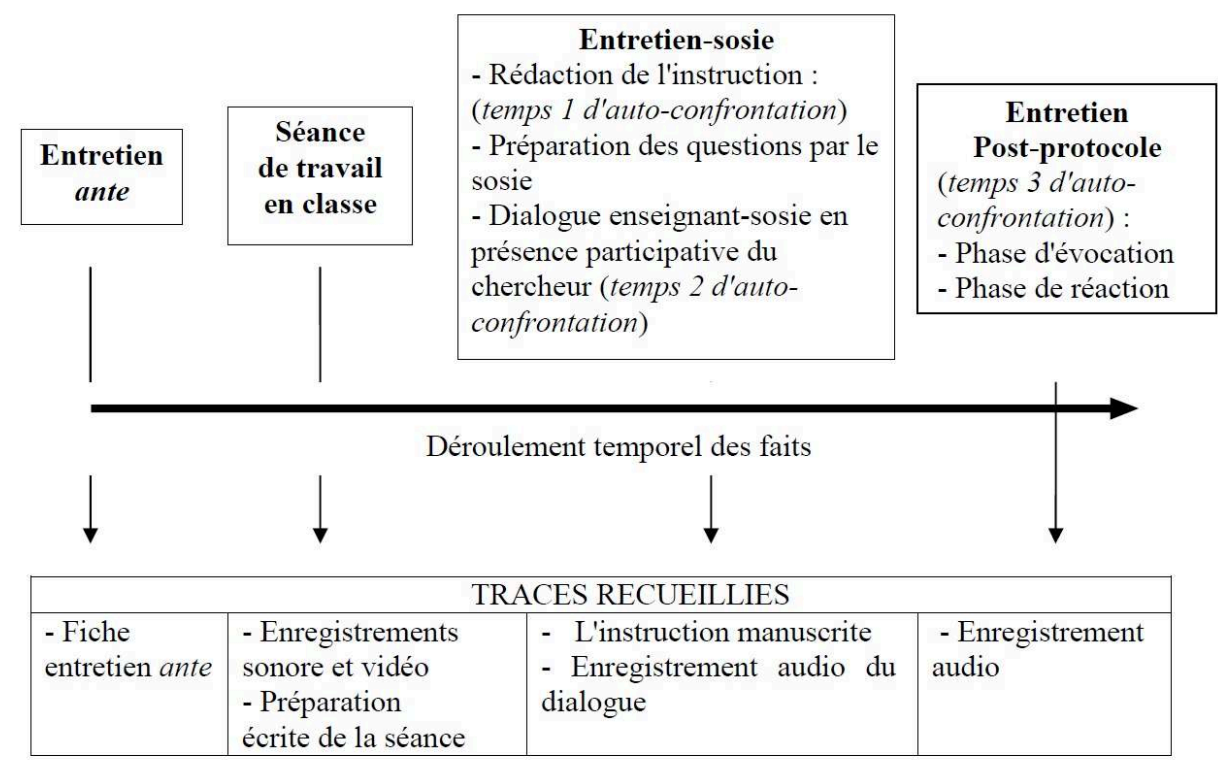

Dans ce protocole, l'entretien ante est bref et consiste en fait à fixer le contrat de recherche avec l'enseignant, notamment au regard de ses prévisions et des savoirs mis à l'étude, la séance qui sera filmée. Le dispositif d'entretiens (composés de l'entretiensosie et de l'entretien post-protocole) se déroule après la séance observée.

Le système de recherche ainsi construit, arrimé au cadre problématique et conceptuel didactique, et alimenté par les apports de la clinique de l'activité, a pour ambition de créer les conditions méthodologiques d'accès au réel de l'activité des enseignants observés et de mettre en enquête l'action d'enseignants d'EPS en milieu difficile en convoquant dans l'analyse les dimensions empêchées de l'activité didactique.

Concrètement, la recherche a concerné 4 duos d'enseignants (enseignant observé et enseignant sosie). Chaque enseignant a été observé lors d'une séance (enregistrement vidéo et audio de l'intégralité de la séance) et a participé à un entretien-sosie après la séance puis à un entretien post-protocole (avec le chercheur).

Les traces recueillies sur lesquelles ont été effectuées les analyses sont de ce fait les enregistrements audio et vidéo des séances ainsi que les enregistrements audio des entretiens-sosie et des entretiens post-protocole. 


\section{Des traces récoltées à la construction de ce qui fait signe}

13 Contrairement aux usages en didactique, les observations en classe n'ont pas un statut de corpus principal. Il n'y a donc pas de chronologie établie dans le recours aux différents corpus de données (Leutenegger, 2003). L'analyse de chacun des cas a été menée sur la base d'allers-retours entre les différentes pièces du corpus, par itérations successives.

L'analyse didactique menée, adossée à la théorie de l'action conjointe en didactique (TACD), se caractérise par une perspective pragmatique (instruire l'action telle qu'elle se déroule) afin d'en décrire la dynamique évolutive qui sous-tend la transmission des savoirs.

Cette posture, ainsi que le principe de triangulation, justifie que nous ayons établi et confronté, à partir de différents moments tirés des diverses traces (vidéo de la séance observée, entretien-sosie, entretien post-protocole) plusieurs scénarios d'explications plausibles aux faits observés, selon une démarche spiralaire dans laquelle de nombreux allers-retours entre les différentes pièces du corpus permettent de réduire, au fur et à mesure, les incertitudes d'interprétation.

La démarche adoptée est illustrée ici à partir de larges extraits de l'analyse d'une des études de cas, celle d'Hélène. Dans les sections qui suivent instruisons le cas par la mise en intrigue (Sensevy, 2010) de différents moments: un qui a trait à une consigne donnée par l'enseignante : « dire j'ai » en réception, l'autre qui s'intéresse à l'évaluation que fait l'enseignante des productions de ses élèves dans l'exercice, avant de mettre au jour les ressorts de l'activité d'Hélène pour saisir dans ce qui est observable ce qui relèverait de son activité didactique empêchée.

\section{Le cas Hélène : séance de volley-ball, classe de $4^{\text {ème }}$}

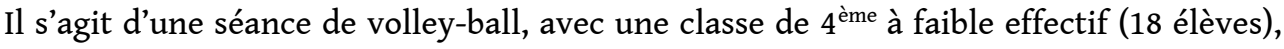
dont le thème est la réception de service. La séance commence par un temps de course qui constitue l'échauffement physique, suivi d'un temps individuel de manipulation de balle (des jonglages). L'échauffement avec ballon se poursuit par un temps de manipulation de balle par deux : travail de la passe et de la manchette (il est utile de rappeler ici que la manchette est le geste technique spécifique de la réception de service et de la défense). Cette première partie de la séance a duré une trentaine de minutes.

Se déroule ensuite, pendant 26 minutes, le corps de la séance qui est composé d'un premier exercice visant le travail du service et de la réception au cours duquel les élèves sont par deux (1 avec 1 ), le deuxième exercice, toujours relatif à la réception de service, met 1 élève face à 3 . La séance se termine par un temps de matchs en 2 contre 2 d'une trentaine de minutes.

La structuration de cette séance laisse penser qu'Hélène a une conception plutôt mécaniste de l'enseignement des sports collectifs se caractérisant par une certaine élémentarisation des savoirs mis à l'étude : les différents gestes techniques (la passe, la manchette, le service) sont appréhendés dans des exercices se rapprochant progressivement du contexte du match (travail individuel, puis par 2, puis en opposition) (Mérand, 1989). 
Dans cet article nous examinons un moment de la séance observée, relatif à un des deux exercices du corps de séance. Nous le décrivons ci-après, à partir de dissonances émergeant de la confrontation des différents corpus.

Le dispositif support des analyses met en jeu 4 élèves dont l'un, le joueur A, est placé, $\mathrm{du}$ côté gauche du filet (Cf. schéma 2). Il fait face à 3 joueurs $\mathrm{D}, \mathrm{C}$ et $\mathrm{B}$, situés dans l'autre camp (1 contre 3$)$. A doit lancer le ballon vers $B$ et $C$, qui doivent se débrouiller pour le renvoyer à $\mathrm{D}$.

Schéma 2. Le dispositif mis en place par Hélène

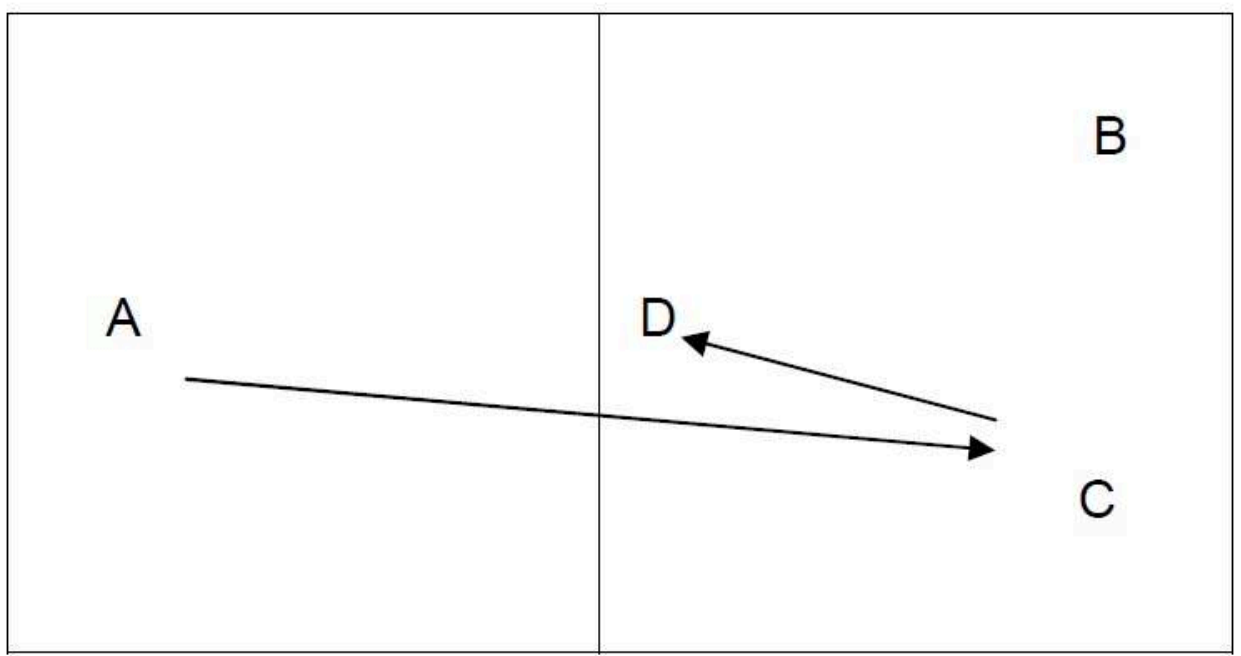

\section{1. L'analyse de l'action de l'enseignante en classe : la question du « j'ai »}

$\mathrm{Au}$ cours de l'entretien-sosie Véronique (le sosie) pose de but en blanc une question surprenante $(\operatorname{tdp} 143 \mathrm{~S})$ : « et quand ils disent «j'ai» tu l'entends le «j'ai»? ». Cette question est surprenante parce que rien n'est évoqué à propos $d u$ «j'ai» par Hélène (le professeur étudié) dans les tours de parole précédents. Comment le sosie est-il amené à aborder ce sujet sans lien apparent avec le déroulement de l'entretien? Il faut revenir à l'information dont dispose Véronique au moment de l'entretien (l'instruction au sosie rédigée par Hélène à l'issue de la séance) pour repérer les données qui sont en sa possession et qui, semble-t-il, sous-tendent la question qu'elle pose. Dans l'instruction ( "bilan » de séance adressé au sosie) Hélène a décrit la tâche des réceptionneurs $B$ et $C$ en ces termes : «les joueurs réagissent en disant «j'ai » et envoient sur $D$ ». La formulation de cette consigne soulève plusieurs questions et suppose quelques clarifications. Elle peut laisser penser que l'enseignante conçoit le fait de dire « $j$ 'ai » comme quelque chose d'assez simple, presque évident, comme s'il suffisait de le demander ("les joueurs réagissent en disant j'ai ») pour que les élèves le fassent. C'est peut-être ce qui a suscité la curiosité ou l'étonnement du sosie. Pourquoi le sosie s'étonnerait de cette conception du « j'ai » en volley-ball ? Qu'est-ce que « dire j'ai » en volley-ball ?

On voit bien ici que pour repérer ce qui fait question dans ces extraits d'entretien il est nécessaire d'analyser le savoir qui est en débat lors de l'entretien-sosie. 


\section{1. 1. Analyse épistémique de l'action « dire j'ai »}

Dire «j'ai » avant de frapper la balle en volley-ball est un aspect emblématique de la pratique sociale de référence. Pour le joueur dans le jeu, dire « j'ai » alors que le ballon est encore en l'air c'est se déterminer et se désigner porteur de balle. Le joueur qui dit « j'ai » signifie à son ou ses partenaires qu'il s'engage et prend la responsabilité de jouer le ballon qui arrive. Cela évite éventuellement que deux joueurs se gênent en intervenant en même temps sur le ballon, cela permet également au(x) partenaire(s) d'être dégagé(s) de cette charge immédiate et de pouvoir s'organiser en se plaçant sur le terrain pour assurer la poursuite de l'action collective. Cette nécessité est renforcée en volley-ball par l'impossibilité de bloquer la balle pour temporiser l'action collective. Dire « j'ai » suppose une prise de responsabilité par le joueur, celle de devenir le porteur de balle. Pour que ce " $j^{\prime} a i »$ remplisse cette fonction dans le jeu, il est nécessaire (au sens de nécessités liées au savoir en jeu) que l'élève :

- anticipe avec assez de justesse le point de chute du ballon pour estimer qu'il peut ou doit le jouer,

- ait une bonne appréciation de son rayon d'action efficace (jusqu'à quelle distance je suis en mesure d'intervenir sur le ballon),

- ose s'engager et prendre le risque en même temps que la responsabilité de l'action,

- réalise les coordinations motrices nécessaires au déplacement et à la frappe du ballon,

- intègre à son action individuelle la possible collaboration de l'action individuelle de ses partenaires et donc s'inscrive dans un projet d'action collectif : une co-activité en résumé.

\section{1. 2. Mise en tension des différentes pièces du corpus pour conduire l'enquête}

La façon dont Hélène décrit la tâche des joueurs $B$ et $C$ dans l'instruction qu'elle adresse à son sosie, se heurte aux données tirées de l'enregistrement de la séance : ce qui s'y est dit et ce que les élèves y ont produit. Qu'avait effectivement dit l'enseignante aux élèves pour leur expliquer l'exercice?

Tableau 1. Extrait de la transcription verbatim de la vidéo

Min 45 : Premier joueur on va l'appeler $A$, deuxième joueur les deux, deux joueurs qui sont au fond ici $B$ et $C$ à peu près au même niveau au fond ils vont être les réceptionneurs, ceux qui réceptionnent soit en manchette soit en passe haute donc ils rattrapent le ballon, que le joueur $\mathrm{A}$ vient de lancer et ils doivent par une passe donner le ballon au joueur D qui est ici dos au filet qui regarde le joueur $\mathrm{B}$ et le joueur $\mathrm{C}$ et il doit récupérer le ballon il le récupère à la main il fait pas de passe il le récupère à la main comme si c'était [?] il rattrape le ballon et il le rend au joueur A qui lance.

Dans la consigne initiale donnée dans le vif de la séance, rien n'apparaît à propos du «j'ai ». L'écart entre ce qui s'est passé dans la séance et ce qu'en dit Hélène au sosie pose question. Qu'est-ce que « dire j'ai » pour Hélène? Revenons à l'entretien-sosie pour voir ce qui s'est échangé entre Hélène et Véronique (à gauche apparaissent les tours de parole, S pour le sosie, $\mathrm{P}$ pour le professeur; nous avons, pour faciliter la lecture, substitué les signes de ponctuations courantes aux codes usuels de transcription des enregistrements audio). 


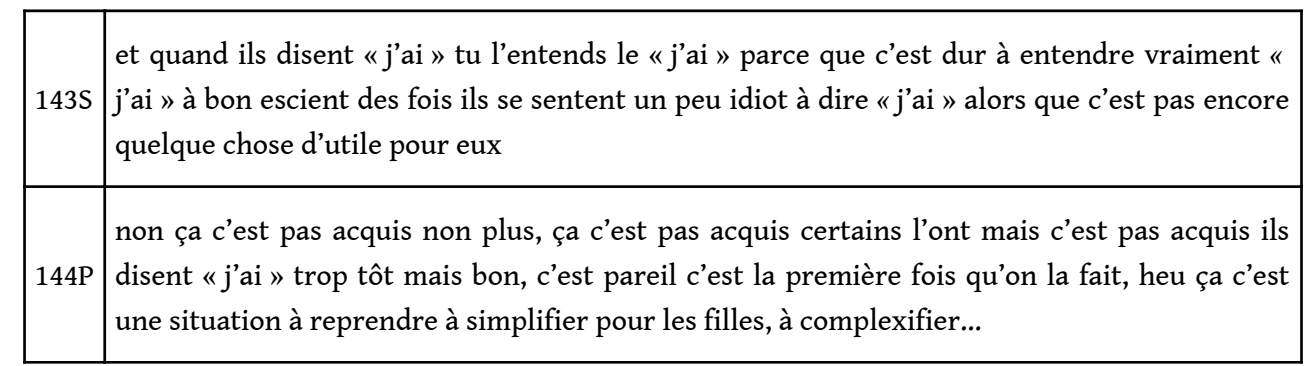

19 Il semble qu'on puisse repérer dans le discours du sosie (143S) des dimensions qui tiennent du savoir tel qu'on l'a brièvement analysé: "Bon escient" renvoie à la pertinence de la décision prise, ou encore "ce n'est pas encore utile pour les élèves » laisse penser que le sosie prend en compte l'évolution de la construction des savoirs qui rendent pertinent et utile ce nouveau savoir pour les élèves. Le caractère plutôt général du discours du sosie permet d'avancer que Véronique semble soumettre à Hélène une discussion plus large: il ne s'agit pas strictement de la séance d'Hélène mais de solliciter le point de vue d'Hélène sur la question, engager un « débat d'école » pourrait dire Clot (2001). En revanche la réponse d'Hélène est plus obscure (144P). On note qu'elle ne rentre pas dans le débat d'école, elle reste arrimée à la séance observée qui s'est déroulée la veille de l'entretien, arrimée à ce qui s'est passé dans cet exercice-là, avec ces élèves-là.

En s'appuyant sur le début de sa réponse, on pourrait considérer qu'Hélène, comme elle l'a écrit au sosie dans l'instruction, pense enseigner (ou avoir enseigné) «dire j'ai » dans l'exercice qu'elle a mis en œuvre. D'ailleurs, elle en évalue le niveau d'acquisition ( "ce n'est pas acquis, ils disent «j'a »i trop tôt»). Mais l'analyse que nous avons faite du dispositif mis en place montre au contraire que le milieu didactique initial proposé aux élèves ne porte pas de façon ciblée sur l'apprentissage de la répartition des rôles porteur de balle/non porteur de balle que « dire j'ai » sollicite.

Revenons à la vidéo pour nous intéresser aux consignes données par Hélène à ses élèves (tableau 1 supra, min 45). Elle procède, au terme de cette première minute de présentation, à la démonstration de l'exercice. Elle assume le rôle du joueur A, celui qui lance le ballon et demande à trois élèves de tenir les rôles de B, C et D (les deux réceptionneurs et $\mathrm{D}$ sur lequel le ballon doit arriver). Voir les échanges à ce propos cidessous (tableau 3):

Tableau 3. Extrait de la transcription verbatim de la vidéo $\min 45-46$

Min 46

Prof: On regarde l'exercice, je suis le joueur A (...) je vais lancer le ballon soit à Samir soit à Stan je leur dis pas le ballon part d'un côté ou de l'autre,

El: Ah vous leur dites pas!

Prof: Non je dis rien pour le moment je lance le ballon et c'est un des deux qui le rattrape si il arrive au milieu à un moment donné il va falloir que vous vous entendiez tous les deux hein donc il va falloir se parler, il va falloir dire c'est moi qui y vais ou c'est toi

Mais en général en volley on dit quoi?

El:j'ai (en cour)

Prof: "J'ai » d'accord et celui qui le dit en premier il y va! 
Notons que rien n'a été dit sur le «j'ai » dans la première partie de la présentation. $C^{\prime}$ 'est au moment de la démonstration que l'enseignante donne de nouvelles indications aux élèves. Elle précise alors la direction du lancer « je vais lancer le ballon soit à Samir soit à Stan je leur dis pas le ballon part d'un côté ou de l'autre ».

Nous considérons que c'est la réaction de l'élève à cette nouvelle information « $E l$ : Ah vous leur dites pas! » qui amène l'enseignante à considérer un état du jeu fortuit, celui où le ballon arriverait entre les deux joueurs, et à introduire alors un nouvel objet dans le milieu : le «j'ai ». L'enseignante le propose comme la solution au problème soulevé lorsque l'élève qui doit jouer le ballon n'est pas clairement désigné par la trajectoire du ballon. Hélène n'avait probablement pas choisi initialement de construire un exercice qui confronte les élèves au problème posé par un ballon arrivant entre les deux réceptionneurs (problème qui nécessite de savoir se répartir les rôles de porteur et non-porteur de balle). Si tel avait été le cas, elle n'aurait pas précisé aux élèves que le lancer doit se faire soit sur l'un ou sur l'autre («soit à Samir soit à Stan») mais, au contraire, ni sur l'un ni sur l'autre des deux réceptionneurs. Cette indication, de nature mésogénétique, aurait été en fait, la seule à-même de garantir la nécessité, pour l'élève en réception, de se désigner réceptionneur en disant " $j$ ' $a i$ ». Le système de contraintes donné par l'enseignante dessine plutôt les contours d'un exercice visant le travail individuel de la réception, voire de la manchette, dans un contexte collectif ( 2 joueurs assurant la réception, plus 1 destinataire $\mathrm{D}$ de la balle). Notons que le fait d'être deux n'est ni un problème particulier compte tenu de la nature du lancer du joueur A (orienté), ni vraiment une ressource (un partenaire), compte tenu de la demande d'atteindre le joueur D directement sans s'aider de son partenaire.

Mais alors, si ce n'était pas son projet didactique initial pourquoi répond-t-elle au sosie dans l'entretien «que ce n'est pas acquis»? Pourquoi l'écrit-elle au sosie dans l'instruction? Que l'enseignante veut-elle ou pense-t-elle enseigner? La fin de l'extrait de l'entretien-sosie relatif à la question $d u$ « j'ai » en réception fait avancer l'enquête :

Tableau 4. Extrait de la transcription verbatim de l'entretien-sosie

\begin{tabular}{|l|l|}
\hline $150 \mathrm{P}$ & $\begin{array}{l}\text { ils disaient « j'ai » trop tôt, ils le disaient pour s'amuser, mais pour moi c'est pas grave dans } \\
\text { le sens où c'est la première fois qu'on fait la situation, que la situation on va la reprendre et } \\
\text { donc quelque part tout ce qui est acquis sur on lance la balle on la réceptionne etc. j'aurai } \\
\text { plus besoin d'y revenir et je reviendrai après sur des «j'ai », sur le placement, à quel moment } \\
\text { plutôt devant, enfin voilà, je reviendrai sur les choses qui sont pas acquises, mais ça } \\
\text { m'inquiète pas ça }\end{array}$ \\
\hline
\end{tabular}

Au tour de parole 150, Hélène met en évidence deux aspects de sa pratique :

- Elle parait concevoir son enseignement en deux temps : d'abord faire apprendre aux élèves le fonctionnement de l'exercice, et dans un deuxième temps s'attacher de plus près aux savoirs plus denses ("j'ai », placement, moment et direction de la réception vers l'avant).

- Il semblerait qu'elle mette au même niveau, ou attribue la même fonction aux consignes expliquant le fonctionnement du dispositif (on lance, on réceptionne) et celles qui ont trait à l'enjeu de savoir («j'ai », le placement).

Ces éléments laissent penser que l'on retrouve ici des résultats ou des constats déjà posés par les sociologues de l'éducation, notamment ceux montrant que le maintien de l'ordre - faire faire l'exercice - prend le pas sur les enjeux didactiques (Kherroubi \& 
Rochex, 2004). L'entretien post-protocole, dernier moment de "réalisation» de son activité par l'enseignant dans notre dispositif de recherche, nous amène néanmoins à développer une autre analyse.

Nous avons soumis l'intégralité du moment de l'entretien-sosie relatif à ce moment de la séance (le " j'ai» en réception) à Hélène lors de l'entretien post-protocole. Nous lui avons présenté le document, en vert étaient surlignés ses propos et en orange ceux de Véronique (le sosie). Les tours de parole distinguent ce que dit Hélène $(\mathrm{P})$ de ce que dis le chercheur (Cher). Hélène lit l'extrait et répond :

Tableau 5. Extrait de la transcription verbatim de l'entretien post-protocole

\begin{tabular}{|l|l|}
\hline $114 \mathrm{P}$ & ouais ouais je remets le truc l'histoire du «j'ai » oui oui je remets \\
\hline $115 \mathrm{Cher}$ & il y a des choses qui te parlent \\
\hline $116 \mathrm{P}$ & ce qui est en vert c'est ce qu'elle a dit elle \\
\hline $117 \mathrm{Cher}$ & non c'est toi \\
\hline $118 \mathrm{P}$ & ah c'est moi ouais (12s) je sais pas (sourire) \\
\hline
\end{tabular}

Autre contexte, autre " réalisation ", c'est en fait dans le discours du sosie qu'Hélène se retrouve et non dans le sien. Perturbée, elle reste longuement pensive avant de se rendre à l'évidence : elle ne comprend plus ce qu'elle a dit au sosie. Elle ne peut plus réagir à l'extrait qu'elle vient de lire. Il semblerait qu'on puisse déceler ici des effets du contexte sur la réalisation de son activité par le sujet. Avançons-le avec la prudence requise au côtoiement d'un champ qui n'est pas le nôtre! Rappelons pour ce faire qu'au moment de l'entretien-sosie, Hélène est très ancrée dans la séance qu'elle vient de vivre (la veille de l'entretien). Cette séance est l'objet du discours formulé dans l'instruction et discuté lors du dialogue, alors qu'au moment de l'entretien postprotocole, la séance est lointaine et l'objet de l'entretien n'est plus la pratique de l'enseignante elle-même mais ce qui s'est dit à propos d'elle. Rappelons aussi que l'interlocuteur n'est plus le sosie (un autre collègue d'EPS), mais le chercheur. Ce contexte de réalisation paraît rendre à Hélène sa propre pratique étrangère, en tout cas ce qu'elle en a dit, qu'elle ne reconnaît d'abord pas. Nous pensons avoir ici accès à son activité empêchée. Ce qui n'a pas pu être dit au sosie, l'est alors : comme un possible enfoui, oublié qui ressurgit par la distance qu'elle prend au regard du contexte de sa pratique quotidienne. Ce nouveau contexte ( $3^{\text {ème }}$ temps d'autoconfrontation dans le protocole de recherche) modifie sensiblement la lecture ou l'analyse qu'Hélène fait de l'objet du débat, en l'occurrence "dire j'ai en réception », puisqu'elle ne re-trouve pas sa pensée dans ses propres mots, mais plutôt dans ceux du sosie. Quel intérêt revêt cette information apportée lors de l'entretien post-protocole?

D'un point de vue méthodologique, l'intérêt d'articuler des voies d'accès directes et indirectes à l'activité pour comprendre l'action didactique observée apparaît manifeste. Ce qui pourrait sembler n'être que contradiction ou dissonance (entre l'observé de la pratique et les échanges avec le sosie ou encore d'un échange à l'autre) devient autant de trappes d'accès au réel de l'activité de l'enseignant qu'il s'agit d'instruire pour expliquer l'action conjointe observée en classe. Nous pensons que le recours au cadre conceptuel de la clinique de l'activité permet d'éclairer des phénomènes que les méthodes en usage dans la TACD ne pouvaient prendre totalement en considération. 
Nous reviendrons sur cette question théorique complexe en fin d'article.

Nous poursuivons donc la mise en intrigue de l'activité de l'enseignante à partir d'un autre faisceau de données, toujours à propos du même exercice de la séance.

\section{2. L'analyse de l'action en classe : la question de ce qu'est une « réception » réussie}

Le bilan de l'exercice qu'énonce Hélène en répondant à une question du sosie relative aux niveaux des élèves en réception de service se heurte aux données tirées de la vidéo de la séance. L'enseignante évalue que " $70 \%$ des élèves arrivaient à envoyer le ballon au passeur ", elle précise que les filles réussissent moins et qu'en revanche, un groupe de garçons sur les deux, parvient même à ce niveau de réussite alors qu'elle avait complexifié la mise en jeu (service et non plus balle lancée).

\section{2. 1. Analyse épistémique du savoir « réceptionner une mise en jeu »}

Avant de poursuivre, il est utile de s'entendre sur ce que sont les niveaux de jeu en réception en volley-ball. Les textes officiels ainsi que la littérature professionnelle définissent globalement 4 niveaux d'habileté dans la réception de service.

- Le premier, le plus faible, concerne les élèves qui ne parviennent pas à se trouver au point de chute du ballon ou qui perdent la balle dès l'impact.

- Le deuxième, correspond aux élèves qui, arrivant tardivement sur le ballon ou n'ayant pas construit toutes les coordinations motrices nécessaires, produisent une réception dite " explosive » : le ballon est fréquemment renvoyé directement dans le camp adverse.

- Le troisième niveau, est celui qui caractérise des élèves parvenant à conserver la balle dans leur camp, mais la qualité de la trajectoire produite (hauteur, direction, vitesse) rend son exploitation difficile par les partenaires de l'équipe.

- Le dernier niveau, concerne les réceptions qui atteignent la zone d'intervention du relayeur : la trajectoire produite est suffisamment haute et orientée vers la zone avant du terrain (proche du filet) pour que ce dernier puisse l'exploiter.

Ainsi, selon la formulation d'Hélène, presque tous les élèves - sur balle lancée et non servie, plus difficile - seraient au niveau le plus expert : celui d'une réception arrivant dans la zone d'intervention du relayeur. Selon elle, quatre d'entre eux sont capables d'atteindre cette zone même sur balle servie.

La lecture de la vidéo de la séance, permet d'établir le niveau des productions effectives des élèves en réception. Pour synthétiser l'analyse, les filles produisent des réponses de niveau 1 ou 2, c'est-à-dire qu'elles ne parviennent pas à initier des trajectoires de balle permettant d'adresser le ballon en direction du relayeur (soit elles n'arrivent pas à être au point de chute de la balle, soit elles la frappent de façon explosive). Les groupes de garçons, envoient le ballon en direction du passeur comme le demande l'enseignante. Néanmoins, les trajectoires produites ne sont, pour la plupart, pas exploitables, car trop basses. Les ballons trop bas, les trajectoires trop tendues, ne sont pas utilisables par le relayeur. Si l'on se place dans une approche fonctionnelle des savoirs mis à l'étude, ces garçons se situent dans le niveau 3 (conservation de la balle dans son camp) et non pas dans le niveau 4 comme le dit l'enseignante. L'écart entre les données vidéo et la description du niveau de jeu des élèves par Hélène dans l'entretien-sosie est confirmé. Cet écart n'est-il qu'un effet de formulation, révèle-t-il une divergence de conception 
des savoirs en jeu dans la réception de service? Ou bien indique-t-il une analyse approximative de ces derniers par Hélène?

\section{2. 2. Indices de l'épistémologie pratique de l'enseignante}

L'analyse de la structuration de la séance peut laisser penser qu'Hélène inscrit ses élèves dans un "contrat didactique de conditionnement » qui révèle en même temps qu'il le met en œuvre, une élémentarisation des savoirs (Brousseau, 1996). L'analyse des productions motrices des élèves qu'elle livre au cours de l'entretien-sosie, semble confirmer une conception mécaniste de l'enseignement du volley-ball (Mérand, 1989) s'appuyant sur des éléments repérables résultant d'un découpage chronologique de l'action (le service, la réception, la passe puis l'attaque). Cette approche, qui s'oppose pour l'auteur à une approche dialectique des sports collectifs, minore l'analyse fonctionnelle des savoirs abordés. Le thème de la séance qu'Hélène annonce aux élèves, et qu'elle indique au sosie dans l'instruction, est la «réception». Celui de la séance précédente était la "mise en jeu». On peut dire que l'approche chronologique et élémentaire des savoirs du volley-ball nous paraît ici confirmée. Elle rend compte de phénomènes transpositifs largement répandus, comme ont pu le montrer des recherches anciennes (Marsenach, 1991). Nous identifions, à ce niveau de l'analyse, des éléments qui tant au niveau des dires que des actions de ce professeur, semblent influencer l'action didactique conjointe que nous observons. Nous les interprétons comme des indices de son épistémologie pratique (Amade-Escot, Amans-Passaga \& Montaud, 2009; Sensevy, 2007). Affinons à ce propos, l'analyse de l'épistémologie pratique de l'enseignante.

Nous tirons du corpus de la vidéo certains points d'appuis. En début de séance, Hélène présente un nouvel exercice qu'elle contextualise par rapport au thème de la leçon (la réception) :

\section{Tableau 6. Extrait du verbatim de séance}

Min 23: Alors! chut! On va voir quelque chose là puisqu'on va travailler un petit peu la réception il faut qu'on voit un petit peu la manchette avant, c'est quoi la manchette? À quel moment on fait une manchette plutôt qu'une passe haute?

Quand vous allez récupérer la balle et que la balle est plus basse que la ligne des épaules et que vous pouvez plus la prendre en haut vous pouvez la prendre donc en manchette.

On perçoit, dans la consigne de l'enseignante, à la fois l'élémentarisation des savoirs (elle isole la manchette) et le fait que la réception soit appréhendée d'un point de vue plus individuel que collectif (qui renvoie à une phase de jeu). La précision qu'Hélène apporte, sous forme de questions posées aux élèves, réintègre néanmoins une dimension plus fonctionnelle de l'objet étudié : la manchette ne se fait pas et ne s'apprend pas pour elle-même, elle (re)devient une réponse technique adaptée à un contexte de jeu (balle haute ou balle basse) qu'il revient aux élèves d'identifier. Dans la tâche dévolue aux élèves, Hélène précise un indice (trait pertinent) pour les aider dans la prise de décision : "la ligne des épaules ».

L'entretien-sosie nous permet d'avancer dans la compréhension de l'épistémologie pratique d'Hélène. En réponse à une question que nous lui posons (en fin d'entretiensosie), Hélène explicite quel est l'objectif de l'exercice de réception évoqué 
précédemment. Sa réponse montre que l'enseignante n'évacue pas la fonction qu'assume la réception de service dans le jeu global : rapprocher le ballon de la future zone d'attaque.

Tableau 7. Extrait de l'entretien-sosie, minute 53

\begin{tabular}{|l|l|}
\hline Cher & {$[. .$.$] là toi tu voulais qu'ils apprennent la manchette enfin la manchette, le renvoi$} \\
\hline 363P & le renvoi vers l'avant c'est à dire passer d'une zone arrière à une zone avant \\
\hline
\end{tabular}

Quelques tours de parole plus loin, Hélène précise sa conception des différentes étapes de la construction par l'élève de la réception. Nous notons qu'elle est proche des analyses usuelles (littérature professionnelle) et intègre à l'analyse du savoir la fonction que remplit la réception de service, en tant que phase de jeu, dans la gestion du rapport de force :

\begin{tabular}{|l|l|}
\hline $409 \mathrm{P}$ & $\begin{array}{l}\text { c'était renvoyer le ballon de la zone arrière vers la zone avant voilà c'était première étape là } \\
\text { ça que c'est évident qu'une deuxième étape c'est renvoyer le ballon correctement la } \\
\text { troisième étape ce sera le la renvoyer le ballon efficace et avoir une action derrière enfin } \\
\text { voilà. }\end{array}$ \\
\hline
\end{tabular}

Tableau 8 : Extrait de l'entretien-sosie, minute 61

Remarquons néanmoins que la définition de la première étape telle que la conçoit Hélène (amener le ballon de la zone arrière vers la zone avant), qui est un thème transversal à tous les sports collectifs (le gagne terrain), est cependant peu spécifié au cadre du volley-ball. C'est la deuxième étape qui semble poser le problème de la qualité de la trajectoire de la balle. Or, la particularité du volley-ball, la «touche » de balle, qui ne permet pas le transport individuel du ballon (le ballon ne peut être bloqué par le porteur de balle), ni la temporisation de l'action par des dribbles (comme en basketball, handball ou encore en football), force à exploiter et à intégrer la verticalité de la trajectoire (en hauteur) au gagne terrain (progression de la balle en avant dans l'aire de jeu). Cette seule réponse de l'enseignante, ne nous permet pas d'établir que les éléments d'analyse du volley-ball que nous venons d'exposer lui sont pour autant étrangers. Le recours à la transcription de séance nous permet d'ailleurs d'abandonner cette hypothèse. En effet la vidéo de la séance montre de façon récurrente que l'enseignante incite les élèves à "monter » les balles (par exemple à la minute 19 "vers le haut! vers le haut! c'est pas assez vers le haut») et donc à explorer la verticalité des trajectoires dont elle ne fait cependant pas état dans sa théorisation de la réception exprimée lors de l'entretien-sosie. Un décalage analogue est observé entre ce qui est perceptible de l'activité de l'enseignante dans le temps privé de la préparation de la séance à partir du dispositif proposé aux élèves (notamment la consigne de renvoyer le ballon sur le joueur D sans plus de précision, ce qui autorise toute sorte de trajectoires lui arrivant dessus) et le commentaire qu'Hélène fait de la production des élèves lors de la démonstration de l'exercice validant une réception haute et vers l'avant comme réponse attendue, sans pour autant exclure les autres possibles.

L'ensemble de ces éléments tirés des différents corpus nous permet de conclure que la conception, ou l'épistémologie pratique d'Hélène ne justifie pas (ou n'explique pas) 
l'évaluation décalée qu'elle fait de ses élèves en réception (Tdp. 123P de l'entretien sosie : avec la mise en jeu de type touche on va dire que $70 \%$ arrivaient à renvoyer le ballon au passeur, à peu près).

Ne pouvons-nous pas alors postuler, qu' au fil du temps passé à exercer en milieu difficile, les possibles abandonnés et les pré-occupations constituant le réel de l'activité de l'enseignante (au sens de Clot, 1999) expliquent cet écart entre ce qui est énoncé dans les entretiens et ce qui se trouve nuancé par ce qui est activé in situ dans l'action conjointe ? C'est ici que l'on peut prendre la mesure de l'épistémologie pratique de ce professeur, au cœur de laquelle restent toujours vifs les possibles de l'activité, comme le met en évidence la poursuite de l'enquête présentée ci-après.

\section{3. Accéder aux ressorts de l'activité didactique empêchée d'Hélène pour comprendre l'action didactique conjointe en classe}

31 La notion « d'activité didactique empêchée » est une construction théorique. Il ne s'agit pas de la rabattre sur des entraves à l'action, des empêchements au sens commun du terme. Dans cette création de concept nous retenons de Clot (1999) que l'activité empêchée n'est pas un dysfonctionnement mais qu'elle est inhérente au régime ordinaire de l'activité de l'homme au travail. Notre apport est de spécifier cette perspective théorique en pointant ce qui dans l'activité de l'enseignant a trait aux enjeux didactiques. Nous ne considérons pas en effet - comme nous l'attribuent, sur la base d'une lecture trop rapide de nos travaux, certains cliniciens de l'activité enseignante (Ruelland-Roger, Moro, \& Matos, 2011) - que ces « empêchements (ici au sens commun du terme) viendraient faire obstacle, dans des situations d'enseignements dégradées, à la mise en œuvre d'une activité didactique. Tout au contraire, nous défendons ci-après la thèse - à partir d'extraits qui, à première vue, pourraient sembler déconnectés de l'analyse didactique des deux objets d'enseignement concernés par les dispositifs mis en place par Hélène ( « dire j'ai », « réceptionner ») - que le lien entre le développement du réel de l'activité (et sa naturalisation au regard du milieu d'exercice) et l'action observable doit être analysé comme la résolution momentanée des conflits du réel qui se jouent entre tous les possibles.

\section{3. 1. L'action de l'enseignante : de l'obéissance à « faire faire »}

\section{3. 1. 1. L'enseignante contrôle sa classe}

Pour Hélène, qui le dit à Véronique le lendemain de la séance lors de l'entretien-sosie (Tdp. 84P) : "sur la séance il y a pratiquement eu aucun problème, pratiquement aucun». Pourtant l'analyse de la vidéo met en évidence un incident avec un groupe de garçons (lié à l'arbitrage du match) qui a monopolisé l'enseignante pendant plus d'un quart d'heure. Qu'est-ce qui amène Hélène à banaliser cet événement, jusqu'à presque l'ignorer?

En fait, Hélène évalue cet épisode comme plutôt positif en ce qu'il révèle son efficacité dans le contrôle de la classe. En effet, elle considère parvenir à gagner des paris risqués : celui d'obtenir des garçons perturbateurs qu'ils s'asseyent avant qu'elle ne les autorise à reprendre le cours du match ( $P$ : «Assieds-toi Sofiane s'il te plait, non mais attends je vais le répéter trois fois là! Assieds-toi s'il te plaît. Samir et Stanislass j'ai dit la même chose, on s'assoit!! Merci», transcription de séance min.71). Ou encore, quatre minutes 
plus tard, lorsque les mêmes élèves, restés assis, obtempèrent à l'injonction d'Hélène (min 75) : $P:$ "On recommence! zéro zéro, c'est moi qui arbitre, tout commentaire entraîne un point pour l'adversaire, est-ce que c'est clair? Salimou est-ce que c'est clair? Sofiane! je parle! en général tu me regardes. Est-ce que c'est clair?». Les images montrent qu'Hélène a gardé le contrôle des élèves turbulents et de l'ensemble du groupe classe, alors que la situation était tendue voire même sur « le fil du rasoir ».

\section{3. 1. 2. Du contrôle de la classe à la direction de l'étude}

Au-delà de ces observations, l'entretien-sosie permet de noter que cette réalité, « tenir sa classe " (Saujat, 2004) offre d'autres bénéfices à Hélène que le seul sentiment de réussite. Il semble que cela lui ouvre des possibles et d'une certaine manière libère son action :

Tableau 9. Extraits de l'entretien-sosie, minutes 4 et 5

\begin{tabular}{|l|l|l|}
\hline $41 \mathrm{P}$ & $\begin{array}{l}\text { c'est une classe si tu la tiens pas elle part de suite [...] voilà et heu mais si tu les tiens ils ne } \\
\text { font que ce que tu dis }\end{array}$ \\
\hline $44 \mathrm{~S}$ & {$[. .$.$] mais à condition d'être très directive$} \\
\hline $45 \mathrm{P}$ & $\begin{array}{l}\text { exactement, mais... directif oui mais juste et avec beaucoup de dialogue, beaucoup de } \\
\text { discussion mais par contre oui très directif }\end{array}$ \\
\hline
\end{tabular}

Pour Hélène, tenir sa classe lui permet d'obtenir de ses élèves qu'ils fassent ce qu'elle leur demande. On note qu'Hélène, accrédite l'analyse que fait le sosie de son mode d'intervention auprès des élèves, elle admet globalement le qualificatif de "directif " (Tdp. 45S). Cette conviction, enracinée dans le terrain de la conduite pédagogique de la classe, l'amène aussi peut-être à affirmer à d'autres moments de l'entretien-sosie que ses élèves "respectent les consignes» (Tdp. 57P), c'est-à-dire qu'ils effectuent la tâche prescrite.

L'entretien se poursuit, les questions du sosie conduisent l'enseignante à se positionner sur la teneur des cours d'EPS: pas de défoulement des élèves et pas de dimension ludique dans le travail, en EPS « on apprend des choses » (Tdp 55P) dit Hélène qui poursuit en explicitant les conditions à réunir pour que le travail puisse être fait par les élèves. Hélène s'est donc expliqué sur ce qu'elle met en œuvre pour que ses élèves apprennent, à la fois en faisant référence à l'un des exercices mis en place dans la séance observée, mais aussi, à partir de considérations plus générales. On peut dire que les thèmes abordés dans les échanges entre le sosie et l'enseignante au cours de ces tours de parole, nous paraissent avoir trait au versant didactique de l'activité d'Hélène. À ce moment du dialogue, alors que le sosie, comme dans l'extrait précédent relatif au contrôle de la classe (tableau 9, Tdp 44S) revient sur l'idée d'attribuer l'efficacité de la conduite de l'étude à la directivité d'Hélène, l'enseignante signifie alors son désaccord avec ce qualificatif. Hélène ne se re-trouve pas dans le regard porté par le sosie sur son activité (Tdp 86P, minute 11 de l'entretien sosie: C'est pas directif, j'aime pas ce mot c'est pas directif on a des routines de travail mais les routines c'est ce qui leur permet d'apprendre »). Hélène parle de "routines " plutôt que de directivité, et établit un lien entre la façon de procéder et le fait de pouvoir faire apprendre les élèves. Autant elle accréditait d'être qualifiée de «directive » pour le contrôle des élèves et de la classe, autant cet attribut ne 
lui convient plus lorsqu'elle revient sur son activité didactique dans ce $2^{\text {ème }}$ temps d'autoconfrontation que constitue l'entretien-sosie. Véronique perçoit-elle une certaine équivalence, ou une proximité, entre les deux versants (conduite de la classe et direction d'étude) dans l'activité de l'enseignante qui l'amène à parler, dans les deux cas, de mode directif? Y aurait-il interférence entre les deux versants de l'activité professorale d'Hélène tel que lui indiquent ou lui suggèrent les remarques du sosie?

Pour instruire cette question, il nous faut revenir sur les techniques mésogénétiques d'Hélène. Comment l'enseignante définit-elle le jeu qu'elle propose aux élèves, qu'elle rôle et quelle fonction assume les consignes données par l'enseignante.

\section{3. 1. 3. Règle de l'exercice, règle constitutive du jeu}

Nous avons vu qu'Hélène paraît dissocier deux temps dans le travail proposé aux élèves. Et peut-être alors aussi deux types d'enjeux d'apprentissage. Un premier serait d'acquérir les règles de déroulement de l'exercice: "on lance la balle», "on la réceptionne", ce qui laisse penser que, d'une certaine manière, les consignes ne semblent pas avoir la fonction d'orienter l'activité de l'élève, ni même de créer des " causes d'apprentissage " (au sens de Brousseau, 1996). Elles sont presque à considérer pour elles-mêmes. En revanche, la consigne qu'elle donne à propos du "dire j'ai », véhicule d'autres savoirs, puisque l'enseignante pense y revenir dans un deuxième temps, qui ne se confond pas avec le fait de dire " $j$ 'ai »-pris ici dans cette tâche, au même niveau que la demande de lancer le ballon au lieu de servir. Se confirme donc un malentendu entre Hélène et Véronique. "Dire j'ai à bon escient » par rapport à l'état du jeu comme le posait le sosie dans l'entretien, ne semble pas être la préoccupation première (ou précise) d'Hélène. En effet, nous avons vu qu'il n'est pas évident, tant dans le dispositif que dans ce qu'en dit ou écrit l'enseignante, que l'on puisse considérer la présence de savoirs masquée derrière cette demande (ou plutôt cette suggestion) de dire « j'ai ».

Pour trancher cette question d'interprétation, nous avons posé la question du statut donné au "j'ai", dans les dispositifs qu'elle met en place à Hélène au cours de l'entretien post-protocole :

Tableau 10. Extrait de l'entretien post-protocole, minute 16

\begin{tabular}{|l|l|}
\hline $145 \mathrm{Cher}$ & $\begin{array}{l}\text { quand tu dis « une fois qu'ils auront acquis le lancer et la réception je reviendrais sur ce } \\
\text { qui n'est pas acquis », alors quelle fonction toi tu donnes au «j'ai » à la fois dans ta } \\
\text { situation et dans l'apprentissage? }\end{array}$ \\
\hline $146 \mathrm{P}$ & heu, quelle fonction je donne au « j'ai », je sais pas trop \\
\hline
\end{tabular}

Hélène ne parvient pas à redonner du sens au choix qu'elle a fait dans la situation en question, "elle ne sait pas trop" pourquoi et dans quel but elle a introduit cette demande.

Nous lui exposons nos pistes d'analyses, celles que nous étions en mesure de livrer à ce moment du traitement du corpus encore bien incomplet : 
Tableau 11. Extrait de l'entretien post-protocole, minute 17

\begin{tabular}{|l|l|}
\hline 159 Cher & $\begin{array}{l}\text { mais toi là tu le places au même niveau quasiment que les consignes qui sont un peu de } \\
\text { l'ordre de l'organisation c'est à dire que }\end{array}$ \\
\hline $160 \mathrm{P}$ & oui \\
\hline 161 Cher & je me pose la question \\
\hline $162 \mathrm{P}$ & oui mais c'est ça \\
\hline 163 Cher & $\begin{array}{l}\text { c'est à dire que ça devient c'est une consigne tu as donné cette consigne là ça devient } \\
\text { une consigne }\end{array}$ \\
\hline $164 \mathrm{P}$ & Oui \\
\hline $165 \mathrm{Cher}:$ & $\begin{array}{l}\text { et ça perd pour l'instant parce qu'après tu vas y revenir mais ça perd pour l'instant dans } \\
\text { un premier temps j'ai l'impression son statut de repère d'apprentissage }\end{array}$ \\
\hline
\end{tabular}

L'échange se poursuit, elle finit par cette conclusion qui accrédite notre intuition de l'époque :

Tableau 12. Extrait de l'entretien post-protocole, minute 21

\begin{tabular}{|l|l|}
\hline $204 \mathrm{P}$ & $\begin{array}{l}\text { Oui oui mais je vois je vois, non mais c'est clair, je crois que c'est exactement ça je base } \\
\text { davantage mon travail d'apprentissage, je les mets dans une organisation où ils } \\
\text { apprennent }\end{array}$ \\
\hline $205 \mathrm{Cher}$ & Hum \\
\hline $206 \mathrm{P}$ & mais ils ont pas de choix à faire pour apprendre \\
\hline 207 Cher & Ouais \\
\hline $208 \mathrm{P}$ & $\begin{array}{l}\text { alors est-ce que derrière tout ça ils sont pas acteurs, j'en sais rien...pourtant ils } \\
\text { apprennent quand même! }\end{array}$ \\
\hline
\end{tabular}

Hélène continue la réflexion amorcée les tours de parole précédents. Elle reformule la conclusion qu'elle tire de ce qu'elle vient de réaliser de son fonctionnement d'enseignante, en prenant en compte cette fois ce que cela implique sur l'activité générée chez les élèves :

Tableau 13. Extrait de l'entretien post-protocole, minute 22

\begin{tabular}{|l|l|}
\hline $212 \mathrm{P}$ & $\begin{array}{l}\text { oui oui, non mais j'ai bien compris, je les mets dans une situation entre guillemets c'est } \\
\text { c'est du coton quoi ils ont tous un rôle tout est précis tout est bien cadré tout est hyper } \\
\text { organisé entre guillemets et ils sont quelque part ils sont limité un rôle unique quoi }\end{array}$ \\
\hline 213 Cher & Hum \\
\hline $214 \mathrm{P}$ & $\begin{array}{l}\text { donc ils ont pas à se déterminer sur un ou deux rôles dans l'apprentissage et c'est vrai... } \\
\text { alors...pourquoi je fais ça...je sais pas... }\end{array}$ \\
\hline
\end{tabular}




\begin{tabular}{|l|l|}
\hline $\begin{array}{l}215 \mathrm{Cher} \\
\mathrm{r}\end{array}$ & mais moi je pense que ça peut être \\
\hline $216 \mathrm{P}$ & $\begin{array}{l}\text { est-ce que c'est parce que justement si je les laisse trop ça peut partir dans tous les sens } \\
\mathrm{j} \text { 'en sais rien...est-ce que c'est une peur ? j'en sais rien... }\end{array}$ \\
\hline
\end{tabular}

À quel «rôle unique » Hélène cantonne-t-elle ses élèves? En revenant au verbatim de l'entretien-sosie, il apparait qu'elle y exprime sa conviction par deux fois (307P) : « oui et puis en plus ce sont des enfants qui sont dans l'action», (338P) : «je pense vraiment que c'est des élèves des gamins qui ont peu d'abstraction et qui n'apprennent que dans l'action». Être dans l'action semble considéré par l'enseignante comme le rapport au monde de ces élèves et comme le moyen d'apprentissage privilégié. Nous avons pu noter également qu'elle considère que ses élèves n'ont pas ou peu de capacité d'analyse (ce qui est largement répandu dans les discours pédagogiques les plus courants sur les milieux difficiles), qu'ils connaissent peu leurs corps, que parmi eux, les filles surtout, ont assez peu envie de travailler. Ces constats, renouvelés depuis 10 ans qu'Hélène enseigne en milieux dits difficiles, fondent probablement en partie la théorie d'action qu'elle a bâtie pour travailler et réussir à travailler dans ces contextes. Ses élèves apprennent donc par l'action et cette croyance, cette conviction conduit Hélène à abandonner d'autres outils possibles. Par exemple, les données d'observation vidéo de la séance, soulignent que les techniques mésogénétiques (les critères de réussites, les consignes constitutives $\mathrm{du}$ jeu) sont comme détournées par l'enseignante. Comme le pointaient d'autres didacticiens (Peltier-Barbier, 2004) tout semble se passer comme si, du fait du développement de son activité en contexte difficile, ces consignes n'ont comme fonction que celle de faire faire la tâche aux élèves. Parvenir à «faire faire » semble prendre le pas sur parvenir à faire apprendre (Rochex, 1997) ce qui peut renforcer ou générer les malentendus observés par Butlen, Peltier-Barbier, et Pezard (2002) et Bautier et Rochex (1997). Il semblerait que nous retrouvions ici la "pacification première » regrettée par Davisse (1996), où le mode directif de la conduite de la classe, devient un véritable préalable à la centration sur les apprentissages fondamentaux de la discipline. Nous pensons cependant que ce n'est pas tout à fait le cas. Car, les ressorts de l'activité didactique empêchée de cette enseignante, qui œuvrent dans la réalité de son action didactique sont plus complexes qu'une simple approche en deux temps. Il semble bien en effet, que la difficulté du public scolaire pèse sur le souci du «maintien de l'ordre » dans la classe comme le développe van Zanten (2001), et que l'obtention de l'obéissance des élèves par l'exercice du pouvoir et non de l'autorité (Arendt, 1972) devienne l'élément impressionnant (au sens photographique du terme) la lecture des événements de classe qui contraint l'activité didactique d'Hélène. Néanmoins, on a pu noter que, si ce souci d'ordre aboutit à la mise en œuvre de dispositifs très fermés, comme l'enseignante le pointe elle-même dans l'entretien post-protocole, les cantonnant à un rôle unique autour d'enjeux de savoir élémentarisés, on a pu toutefois observer que c'est aussi la compétence didactique de cette enseignante qui continue d'œuvrer lorsqu'elle intervient pour réintroduire des objets symboliques dans le milieu (le «j'ai» contextualisé à la perspective d'une coordination d'action entre réceptionneurs, la hauteur des trajectoires nécessaire à la réussite de la tâche). Cette "efficacité malgré tout» témoigne, comme le dirait Clot (1999), des tentatives de récupérations qui sont au cœur de l'activité au travail, et qui ici (comme dans les autres 
cas étudiés dans cette recherche) crée des conditions d'apprentissage. C'est aussi ce qui permet à Hélène - dans le temps long du cycle ou de l'année - de constater que ses élèves « apprennent quand même ».

\section{3. 2. L'action de l'enseignante : un scénario explicatif plausible}

$\mathrm{Au}$ terme de cette étude singulière, nous pensons pouvoir dire que les "préoccupations » en tension chez cette enseignante l'amène à agir « dans une interférence des intentions » (Clot, 1999) expliquant l'action didactique observée :

- Préoccupée d'obtenir et de maintenir la classe en bon ordre, Hélène, au sein de l'équipe EPS, a développé un usage professionnel favorisant, ou créant les conditions d'une obéissance des élèves : un travail routinisé.

- Analysé d'un point de vue didactique, «le cadre qui leur permet d'apprendre » tel que le définit Hélène, se caractérise par des dispositifs simples, mettant en scène des savoirs élémentarisés.

- Cette naturalisation de l'agir produite par le contexte de travail semble peser sur l'analyse didactique que fait l'enseignante des savoirs à enseigner en volley-ball. L'abandon de certains possibles (capacité d'abstraction des élèves par exemple) conduit Hélène à redéfinir les formes d'étude possibles actualisant un ancrage devenu nécessaire dans le concret. L'activité didactique empêchée de cette enseignante semble la contraindre à une analyse mécaniste des sports collectifs, plus compatible avec des exercices simplificateurs qui n'exigent des élèves que de "faire ce qui est demandé».

- Mais les possibles abandonnés (l'analyse fonctionnelle des savoirs, la réflexion des élèves sur leur pratique) continuent néanmoins d'œuvrer et sont au principe de la compétence didactique d'Hélène.

41 Ces constats renvoient aux éléments centraux du concept « d'activité empêchée », dont Clot (1999) montre qu'ils sont au cœur de la dynamique de développement de l'activité. L'analyse que nous menons à partir de l'articulation des cadres théoriques de la didactique et de la clinique de l'activité, soutient une interprétation permettant de mettre au jour, comment dans le vif de la séance, dans l'action conjointe avec ses élèves, Hélène réussit «malgré tout » à ré-introduire certains objets pertinents dans le milieu de l'étude.

Nous voulons souligner ici, comment le système de recherche mis en place permet de voir ce qui ne se fait pas, mais qui pourtant reste vif dans l'intention didactique de l'enseignant et que l'on n'aurait pas décelé si l'on s'en était tenu au dispositif usuel d'observation « du » didactique ordinaire. Le développement de l'activité se loge aussi dans ces espaces que l'action n'occupe pas. C'est dans l'analyse fine des distorsions, des possibles contraints, des entrelacs des raisons d'agir, que nous avons tenté de décrire de nouveaux scénarios du didactique pour comprendre l'action des enseignants de milieux difficiles.

\section{Du singulier de chacun des cas à « l'activité didactique empêchée »}

42 Les quatre cas analysés, illustrés ici par le cas d'Hélène, nous permettent de pointer que les difficultés objectives (le comportement des élèves, leur attitude plus ou moins scolaire, ou plus ou moins disciplinée) qui occurrent dans la séance, font évoluer, au fil 
du temps, le réel de l'activité (le possible et l'impossible) des enseignants observés. Nous avons mis en évidence la réalité d'une activité contrariée, empêchée, sans cesse à rattraper, qui dessine la scène de l'action de l'enseignant en classe comme une coexistence de préoccupations multiples qu'il nous paraît difficile de qualifier de concurrentes ou de dilemmatiques. Ni vraiment en concurrence, ni réellement dilemme, les préoccupations multiples dans lesquelles les enseignants tissent leur activité de travail, se mêlent, s'imbriquent, et, contraignent l'action didactique qui finalement, malgré tout, vainc! Par cette formule nous souhaitons pointer l'apport de notre contribution en terme d'étude du didactique.

Ainsi, si notre étude confirme la complexité des motifs d'agir professoraux, elle met au jour par la convocation dans l'analyse des entrelacs des raisons d'agir, que la résolution des conflits du réel n'emprunte pas la voie de l'abandon strict, ou de la hiérarchisation nette, des préoccupations identifiées. L'action didactique observable, qui advient au cœur de possibles contraints, poursuit un « objet d'horizon » lointain dépassant l'action elle-même tout en lui donnant sens, celui de rendre possible l'aboutissement de l'enjeu de savoir. Nous pouvons dire qu'il ne s'agit pas de renoncement, bien au contraire. Nous pensons avoir établi, en ayant pris au sérieux l'épaisseur théorique du concept d'activité empêchée que nous reconfigurons en terme d'activité didactique empêchée, qu'il rend compte de ce qui se joue dans le travail des professeurs aux prises avec un environnement réel (les contextes observés, les élèves auxquels ils enseignent) et symbolique (les discours pédagogiques, médiatiques, professionnels, institutionnels) qui contraint leur action didactique in situ.

\section{1. Un topos surplombant exacerbé}

L'abandon de possibles, qui trouve son origine dans la naturalisation d'événements se produisant dans le quotidien de travail (l'inattention des élèves, la contestation ou $a$ contrario les demandes qu'ils formulent), mais aussi dans les représentations les plus répandues caractérisant les élèves de milieu difficile, conduisent les enseignants à reorienter et à re-fonder le sens qu'ils donnent à certains gestes qui pourraient contribuer à la dévolution du jeu d'apprentissage. La capacité d'attention, le sérieux de l'investissement dans les tâches, le respect strict des consignes constitutives du jeu, la tenue de fiche de score ou de fiche d'observation sur les productions de leur camarades, l'intérêt pour le travail rigoureux et exigeant sont autant de dispositions dont les enseignants ne pensent plus leurs élèves capables, alors même que nous avons pu observer des élèves qui sont malgré tout attentifs, engagés dans les tâches, soucieux de comprendre les raisons de leurs actions. C'est dans ces croyances que s'enracine l'abandon de possibles topogénétiques qui pourraient permettre de maintenir certaines conditions de dévolution. Le repérage par les élèves des traits pertinents relatifs aux savoirs en jeu grâce à l'observation de critères de réalisation, ou encore par la notification des scores obtenus en lien avec des critères de réussite ciblant les réponses prometteuses, n'est alors plus mis en œuvre par les enseignants. Ces derniers gardent la maitrise des moyens de validation et de sélection des réponses qui vont dans le sens, non pas forcément de l'acquisition souhaitée, mais du savoir potentiellement en jeu. Nous avons vu au travers de ce cas que, dans l'action conjointe, les interventions professorales mettent en lumière le savoir visé alors même que les milieux didactiques initiaux, du fait du développement de l'activité didactique empêchée, ne dessinent plus que des contours assez lâches. Les élèves sont ainsi dirigés vers une effectuation 
versant du côté du «faire ", et l'enseignant, contraint d'occuper la place qu'il a retirée à l'élève, semble ne produire que des énoncés évaluatifs ou de validation. Les possibilités de voir les élèves s'engager proprio motu dans le travail sont réduites et renforcent, peut-être en retour, les signes (attention diffuse, impatience) qui en sont à l'origine. Et pourtant, dans tous les cas, nous avons observé que les professeurs développaient une intense activité de rattrapage des situations dans lesquelles ils agissent. Dans l'action conjointe ils réintroduisent ainsi, certains objets symboliques " faisant milieu " (Schubauer-Leoni, Leutenegger, Ligozat \& Fluckiger, 2007). Ce qui est dit par les enseignants, dans le vif de la séance, s'attache aux traits pertinents de ce qu'il y a à produire dans les dispositifs, ou encore, semble participer à la construction conjointe d'une référence afin d'aider les élèves (certains d'entre eux) à repérer ce qui est attendu.

Ces constats établissent que très souvent l'action de l'enseignant se fait "en main haute » dirait Schubauer-Leoni (2008). Néanmoins, si le topos surplombant adopté par ces enseignants ne favorise pas la dévolution du jeu, nous avons pu constater que ces élèves, soient disant peu scolaires, s'engagent in fine dans le travail demandé par les enseignants alors que l'on a toutes les raisons de penser que beaucoup d'entre eux ignorent pourquoi ils font l'exercice et ce qu'il y a à apprendre en le faisant. Nous considérons que le jeu des élèves doit cependant bien rencontrer celui du professeur, puisque les élèves, dont les raisons de faire nous paraissent relever de la docilité, poursuivent néanmoins le jeu engagé et y opèrent quelques apprentissages.

\section{2. Retour sur nos analyses et leurs présupposés théoriques}

Au terme de notre étude nous estimons renforcée l'idée de devoir articuler les voies d'accès directes et indirectes à l'analyse de l'action didactique impulsée par Leutenegger et Schubauer-Leoni (2002) lorsque ces auteures proposent d'encadrer les séances observées par des entretiens.

Nous avons pu mesurer l'intérêt de s'appuyer à la fois sur l'analyse du dispositif mis à l'étude et sur la gestion qu'en fait l'enseignant dans l'action conjointe pour comprendre la dynamique du processus, tout en ne perdant pas de vue qu'il est insuffisant de prendre en compte uniquement ce qui est observable pour rendre compte des ressorts de l'action didactique. L'activité didactique empêchée qui scelle théoriquement le rapport entre ce qui est fait et ce qui n'est pas fait, qui n'est de ce fait pas observable, mais pour autant accessible via une approche dialogique ouvre de nouvelles perspectives pour l'étude des pratiques didactiques ordinaires. Les analyses effectuées qui confrontent la robustesse de ce lien théorique à l'empirie, contribuent nous semblet-il à alimenter la réflexion à propos du travail professoral. La portée heuristique de la TACD est de rendre compte des phénomènes transpositifs à l'œuvre en classe ordinaire en développant des analyses ascendantes au carrefour d'approches épistémologiques et interactionnelles. Dans l'exploration de ces phénomènes, le recours à la clinique de l'activité permet, en convoquant dans l'analyse une théorie du sujet, de prendre en compte différents registres de préoccupations en lien avec les différentes temporalités dans lesquelles se tisse l'activité professorale même lorsqu'elle est observée à l'aune du temps court que constitue l'échelle d'une séance. 


\section{BIBLIOGRAPHIE}

Amade-Escot C., Amans-Passaga, C. \& Montaud D. (2010). Les savoirs mobilisés dans l'action didactique par les intervenants en activités physiques et sportives : un entrelacs de processus épistémiques et institutionnels. Sciences de la Société, 77, 43-62.

Arendt, H. (1972). La crise de la culture. Gallimard. Folio essais.

Bautier, É. \& Rochex, J.Y. (1997). Apprendre : des malentendus qui font la différence. In J.P. Terrail (Ed). La scolarisation de la France : Critique de l'état des lieux (pp. 105-122). Paris : La dispute.

Brousseau, G. (1988). Le contrat didactique : le milieu. Recherche en Didactique des Mathématiques, vol. 9, $n^{\circ} 3,309-336$.

Brousseau, G. (1996). L'enseignant dans la théorie des situations didactiques. In R. Noirfalisse et M-J. Perrin-Glorian (Eds), Actes de la VIIIème école d'été de didactique des mathématiques (pp. 3-46). Clermont Ferrand.

Butlen, D. ; Peltier-Barbier, M-L. \& Pezart M. (2002). Nommés en REP, comment font-ils? Pratiques de professeurs d'écoles enseignant les mathématiques en REP . Revue française de pédagogie, 140, 41-52.

Clot, Y. (1999). La fonction psychologique du travail. Paris : PUF, Le travail humain.

Clot, Y. (2001). Éditorial. Education Permanente, 146, 7-16.

Davisse, A. (1996). Repenser l'enseignement. La règle et le sens : le dur métier d'élève. Panoramiques, 26, 103-108.

Kherroubi, M. \& Rochex J.Y. (2002). La recherche en Éducation et les ZEP en France. 1. Politique ZEP, objets, postures et orientations de recherche (note de synthèse). Revue Française de Pédagogie, 140, 103-131.

Kherroubi, M. \& Rochex J.Y. (2004). La recherche en Éducation et les ZEP en France. 2. Apprentissages et exercice professionnels en ZEP : résultats, analyses et interprétations (note de synthèse). Revue Française de Pédagogie, 146, 115-181.

Leutenegger, F. (2003). Étude des interactions didactiques en classe de mathématiques : un prototype méthodologique. Bulletin de Psychologie 56(4) 559-571.

Marsenach, J. (1991). Quelques aspects du fonctionnement de l'enseignement de l'EPS. In J. Marsenach et al. (Eds) EPS : Quel enseignement? (pp. 33-58). Paris : INRP. Collection didactiques des disciplines.

Mérand, R. (1989). La rénovation des contenus d'enseignement : Jeux sportifs collectifs au collège. Revue Française de Pédagogie, 89 ,11-14.

Monnier, N. (2009). L'activité didactique empêchée, entre contraintes et ingéniosité : Étude de cas en Éducation Physique et Sportive en milieu difficile. Thèse de doctorat en Sciences de l'éducation, non publiée, Université de Toulouse, Paul Sabatier, Toulouse, France.

Monnier, N. \& Amade-Escot, C. (2007). L'activité didactique empêchée : Construction méthodologique entre science didactique et science du travail. Communication au Symposium. De l'analyse de l'activité des enseignants débutants à la formation des maîtres : Quelles articulations ? Actes du 
Congrès de l'AREF, Strasbourg, 28-31 Août, retriewed, http://www.congresintaref.org/actes_pdf/ AREF2007_Nathalie_MONNIER_370.pdf

Oddonne, Y. ; Rey, A. \& Briante, G. (1981). Redécouvrir l'expérience ouvrière. Vers une autre psychologie du travail. Paris : Éditions sociales.

Peltier-Barbier M.-L. (2004). Dur d'enseigner en ZEP. Dur pour les élèves, Dur pour les enseignants. Grenoble : Éd. La Pensée sauvage.

Poggi-Combaz, M.P. (2002). Distribution des contenus d'enseignement en EPS au collège selon les caractéristiques sociales du public scolaire : des différences non aléatoires. Revue Française de Pédagogie, 139, 53-69.

Rochex, J.Y. (1997). Les ZEP un bilan décevant. In J.P. Terrail. La scolarisation de la France : Critique de l'état des lieux (pp. 123-140). Paris : La dispute

Ruelland-Roger, D. Moro, T. et Matos S. (2011, mars). « S'expliquer » entre professionnels pour « s'expliquer » avec les didactiques. Communication colloque international INRP, 16, 17 et 18 mars 2011 sur « le travail enseignant au XXI ${ }^{\text {ème }}$ siècle perspectives croisées : didactiques et didactique professionnelle ». www.inrp.fr/archives/colloques/travail-enseignant/contrib/ 104.pdf

Saujat, F. (2004). «L'auto-confrontation croisée » comme milieu de travail sur l'activité enseignante. In Marcel, JF, Les pratiques enseignantes hors de la classe. Paris. L'Harmattan, Savoir et formation.

Schubauer-Leoni, M.L. (2008). La construction de la référence dans l'action conjointe professeurélève. In N. Wallian, MP. Poggi, \& M. Musard (Eds.), Co-construire des savoirs : les métiers de l'intervention par les APSA (pp. 67-86). Besançon : PUFC.

Schubauer-Leoni, M.L. \& Leutenegger, F (2002). Expliquer et comprendre dans une approche clinique/expérimentale du didactique ordinaire. In F. Leutenegger et M. Saada-Robert (Eds). Expliquer et comprendre en sciences de l'éducation. (pp. 227-251). Bruxelles : De boëck, Raisons éducatives.

Schubauer-Leoni, M.L. \& Leutenegger, F. (2003). Didactique comparée. In D. Groux (Fir), S. Perez, L. Porcher, V.D. Rust, N. Tasaki (Eds.). Dictionnaire d'éducation comparée. (pp. 70-74). Paris : L'harmattan, Éducation Comparée.

Schubauer-Leoni, M.L., Leutenegger, F. \& Forget, A. (2007). L'accès aux pratiques de fabrication de traces scripturales convenues aux commencements de la forme scolaire : interrogations théoriques et épistémologiques. Éducation \& Didactique, 1(2), 7-35.

Schubauer-Leoni, M.L., Leutenegger, F., Ligozat, F., \& Fluckiger, A. (2007). Un modèle de l'action conjointe professeur-élèves : les phénomènes didactiques qu'il peut/doit traiter. In G. Sensevy, \& A. Mercier, (Eds.). Agir ensemble. L'action didactique conjointe du professeur et des élèves (pp. 51-91). Rennes : Presses Universitaires.

Sensevy, G. (2007) Des catégories pour décrire et comprendre l'action didactique. In G. Sensevy, et A. Mercier, (Eds.). Agir ensemble. L'action didactique conjointe du professeur et des élèves (pp. 13-49). Rennes : Presses Universitaires.

Sensevy, G. \& Mercier, A. (2007). Agir ensemble : Éléments de théorisation de l'action conjointe du professeur et des élèves. Rennes : PUR.

Sensevy, G. (2010). Esquisse d'une pragmatique didactique. Psychologie de l'interaction, 27-28, 179-223. 
Van Zanten, A. (2001). L'école de la périphérie. Scolarité et ségrégation en banlieue. Paris : PUF.

\section{RÉSUMÉS}

Cet article rend compte d'une recherche réalisée auprès de quatre professeurs d'éducation physique et sportive enseignant en milieu difficile, s'attachant à problématiser et éclairer depuis la didactique - le constat réalisé par de nombreux travaux de sociologie et de sciences de l'éducation: celui d'une minoration des savoirs enseignés au bénéfice des savoir-être et de savoir-faire sociaux dans ces contextes d'enseignement. Pour ce faire le cadre théorique intègre, après les avoir convertis, le concept «d'activité empêchée » et la méthode "d'instruction au sosie " empruntés à la "clinique de l'activité » au sein d'une problématique de recherche didactique. L'articulation de l'analyse de l'action didactique en classe à partir de la vidéo et du recours, par des voies d'accès indirectes, à l'activité empêchée de l'enseignant permet ainsi de mettre au jour les ressorts et les contraintes à l'origine du fonctionnement des systèmes didactiques observés.

Looking at schooling in deprived area, sociologists and pedagogists have pointed out that specific learning achievement might disappear for the benefit of social control under the slogan of the making of citizen through students' self-responsibility and self-awareness. In this thesis we analyse through observation and interviews the difficulties encountered by four PE teachers in maintaining quality content knowledge in deprived area schools. Grounded on the "didactique" approach and using the method of "instruction au sosie", the research highlights the idea that the teacher's didactical activity might be understood as "detained". It points out the tensions and the contradictions that teachers have to face all along the specific knowledge co-construction process engaged with their students.

\section{INDEX}

Mots-clés : didactique comparée, didactique de l'EPS, milieu difficile, action conjointe, activité didactique empêchée, savoirs enseignés, instruction au sosie

Keywords : comparative didactics, PE didactics, deprived schools, joint action, didactical activity as detained, knowledge taught, instruction au sosie

\section{AUTEURS}

\section{NATHALIE MONNIER}

UMR-EFTS, Université de Toulouse 2 - Le Mirail

\section{CHANTAL AMADE-ESCOT}

UMR-EFTS, Université de Toulouse 2 - Le Mirail 